\title{
Equation of state for nuclear matter in relativistic mean-field theory and Maxwellian phase transition to strange quark matter
}

\author{
Grigor Alaverdyan* \\ Yerevan State University \\ A.Manoogyan str. 1, 0025 Yerevan, Armenia \\ E-mail: galaverdyan@ysu.am
}

\begin{abstract}
Equation of state for superdense nuclear matter is considered in the framework of relativistic mean-field theory, when also the scalar-isovector $\delta$-meson effective field is taken into account. Assuming that the transition to the strange quark matter is a usual first-order phase transition described by Maxwells construction, the changes of the parameters of phase transition caused by the presence of $\delta$ - meson field are investigated. To describe a quark phase the advanced version of the MIT bag model is used, in which the interactions between $u, d, s$ quarks are taken into account in the one-gluon exchange approximation. For different values of the bag constant $B$, some series of the equations of state of matter with deconfinement phase transition are constructed. Also the upper bound, $B_{c r}$, corresponding to the unstable state of the infinitizimal quark core in a neutron star is found.
\end{abstract}

8th Conference Quark Confinement and the Hadron Spectrum

September 1-6 2008

Mainz, Germany

\footnotetext{
* Speaker.
} 


\section{Introduction}

Within the framework of Quantum Hadro-Dynamics (QHD) the quantum-theoretical approach allows to adequately enough describe properties both of nuclear matter and finite nuclei, considering them as a system of strongly interacting baryons and mesons. One of the similar theories is the relativistic mean-field theory (RMF) which is effectively used [1]. Inclusion of the scalar-isovector $\delta$-meson in this scheme and investigation of its influence on low density asymmetric nuclear matter properties was realized in Ref.[2-3]. In this paper the EOS of superdense nuclear matter is studied in the framework of RMF approach, the deconfinement phase transition from hadronic matter to quark matter is analyzed, and the role of scalar-isovector $\delta$-meson in this process is investigated.

\section{Deconfinement phase transition parameters}

The relativistic Lagrangian density of an interacting many-particle system consisting of nucleons and $\sigma, \omega, \rho, \delta$-mesons in QHD is given by

$$
\mathscr{L}_{\sigma \omega \rho \delta}\left(\sigma(x), \omega_{\mu}(x), \vec{\rho}_{\mu}(x), \vec{\delta}(x)\right)=\mathscr{L}_{\sigma \omega \rho}\left(\sigma(x), \omega_{\mu}(x), \vec{\rho}_{\mu}(x)\right)-U(\sigma(x))+\mathscr{L}_{\delta}(\vec{\delta}(x)),
$$

where $\mathscr{L}_{\sigma \omega \rho}$ is the linear part of relativistic Lagrangian density without $\delta$-meson field [4],

$$
U(\sigma)=\frac{b}{3} m_{N}\left(g_{\sigma} \sigma\right)^{3}+\frac{c}{4}\left(g_{\sigma} \sigma\right)^{4} \text { and } \mathscr{L}_{\delta}(\overrightarrow{\boldsymbol{\delta}})=g_{\delta} \bar{\psi}_{N} \vec{\tau}_{N} \overrightarrow{\boldsymbol{\delta}} \psi_{N}+\frac{1}{2}\left(\partial_{\mu} \vec{\delta} \partial^{\mu} \vec{\delta}-m_{\delta} \overrightarrow{\boldsymbol{\delta}}^{2}\right) \text { are the } \sigma-
$$

meson self-interaction term and contribution of the $\delta$-meson field, respectively. This Lagrangian density (2.1) contains the meson-nucleon coupling constants, $g_{\sigma}, g_{\omega}, g_{\rho}, g_{\delta}$ and also parameters of $\sigma$-field self-interacting terms, $b$ and $c$. Constants of RMF theory, $a_{i}=\left(g_{i} / m_{i}\right)^{2}(i=\sigma, \omega, \rho, \delta)$ and $b, c$ are numerically determined to reproduce such empirically known characteristics of symmetric nuclear matter at saturation density as the mass of bare nucleons, $m_{N}=938.93 \mathrm{MeV}$, the nucleon effective mass factor, $\gamma=m_{N}^{*} / m_{N}=0.78$, the baryon number density at saturation, $n_{0}=0.153 \mathrm{fm}^{-3}$, the binding energy per baryon, $f_{0}=-16.3 \mathrm{MeV}$, the incompressibility modulus, $K=300 \mathrm{MeV}$, and asymmetry energy $E_{s y m}^{(0)}=32.5 \mathrm{MeV}$.

In this paper $a_{\delta}=2.5 \mathrm{fm}^{2}$ is chosen, according to Ref.[2]. The values of obtained RMF theory parameters are listed in Table 1.

Table 1: The constants of RMF theory with $(\sigma \omega \rho \delta)$ and without $(\sigma \omega \rho) \delta$-meson field.

\begin{tabular}{|c|c|c|c|c|c|c|}
\hline & $a_{\sigma}, \mathrm{fm}^{2}$ & $a_{\omega}, \mathrm{fm}^{2}$ & $a_{\delta}, \mathrm{fm}^{2}$ & $a_{\rho}, \mathrm{fm}^{2}$ & $b, \mathrm{fm}^{-1}$ & $c$ \\
\hline$\sigma \omega \rho$ & 9.154 & 4.828 & 0 & 4.794 & $1.654 \cdot 10^{-2}$ & $1.319 \cdot 10^{-2}$ \\
\hline$\sigma \omega \rho \delta$ & 9.154 & 4.828 & 2.5 & 13.621 & $1.654 \cdot 10^{-2}$ & $1.319 \cdot 10^{-2}$ \\
\hline
\end{tabular}

The results of our analysis show that the scalar - isovector $\delta$-meson field inclusion increases the value of the energy per nucleon, besides, this change is strengthened with the increase of the nuclear matter asymmetry parameter, $\alpha=\left(n_{n}-n_{p}\right) / n$. The $\delta$-field inclusion leads to the increase of the EOS stiffness of nuclear matter due to the splitting of proton and neutron effective masses, and also due to the increase of asymmetry energy (for details see Ref.[5]).

To describe the quark phase an improved version of the MIT bag model is used, in which the interactions between $u, d, s$ quarks inside the bag are taken in a one-gluon exchange approximation [6]. For quark masses we choose $m_{u}=5 \mathrm{MeV}, m_{d}=7 \mathrm{MeV}$ and $m_{s}=150 \mathrm{MeV}$, and $\alpha_{s}=0.5$ 
for the strong interaction constant. Calculations are carried out for different values of the bag parameter $B$ in the range of $60 \leq B \leq 120 \mathrm{MeV} / \mathrm{fm}^{3}$.

Table 2: The Maxwellian phase transition parameters with and without $\delta$-meson field.

\begin{tabular}{|c|c|c|c|c|c|c|c|}
\hline $\begin{array}{c}B \\
\mathrm{MeV} / \mathrm{fm}^{3}\end{array}$ & $\begin{array}{c}n_{N} \\
\mathrm{fm}^{-3}\end{array}$ & $\begin{array}{c}n_{Q} \\
\mathrm{fm}^{-3}\end{array}$ & $\begin{array}{c}P_{0} \\
\mathrm{MeV} / \mathrm{fm}^{3}\end{array}$ & $\begin{array}{c}\varepsilon_{N} \\
\mathrm{MeV} / \mathrm{fm}^{3}\end{array}$ & $\begin{array}{c}\varepsilon_{Q} \\
\mathrm{MeV} / \mathrm{fm}^{3}\end{array}$ & $\lambda$ \\
\hline 60 & $\sigma \omega \rho$ & 0.1220 & 0.2826 & 1.965 & 115.8 & 270.9 & 2.299 \\
\hline 60 & $\sigma \omega \rho \delta$ & 0.1207 & 0.2831 & 2.11 & 114.5 & 271.4 & 2.327 \\
\hline 69.3 & $\sigma \omega \rho$ & 0.246 & 0.3557 & 15.57 & 239.6 & 353.4 & 1.385 \\
\hline 69.3 & $\sigma \omega \rho \delta$ & 0.2241 & 0.3504 & 13.84 & 217.5 & 347.9 & 1.504 \\
\hline 80 & $\sigma \omega \rho$ & 0.3792 & 0.4819 & 48.54 & 384.5 & 501.8 & 1.159 \\
\hline 80 & $\sigma \omega \rho \delta$ & 0.3276 & 0.4525 & 37.95 & 328.8 & 468.6 & 1.278 \\
\hline 100 & $\sigma \omega \rho$ & 0.5506 & 0.7175 & 121.3 & 593.4 & 810 & 1.133 \\
\hline 100 & $\sigma \omega \rho \delta$ & 0.4746 & 0.6497 & 93.30 & 503.3 & 723.5 & 1.213 \\
\hline 120 & $\sigma \omega \rho$ & 0.6512 & 0.8975 & 180.2 & 728.9 & 1073 & 1.18 \\
\hline 120 & $\sigma \omega \rho \delta$ & 0.5729 & 0.8165 & 143.9 & 631.7 & 961.4 & 1.24 \\
\hline
\end{tabular}

We assumed that deconfinement phase transition of nuclear matter into quark matter is a usual first-order phase transition described by Maxwells construction. The Table 2 represents the values of parameters of such phase transition for different values of the bag parameter $B$ with $(\sigma \omega \rho \delta)$ and without $(\sigma \omega \rho)$ the $\delta$-field. In this Table $n_{N}$ and $n_{Q}$ are the baryon number densities at transition point of nuclear and quark phases, respectively, $\varepsilon_{N}$ and $\varepsilon_{Q}$ are the energy densities, $P_{0}$ is the phase transition pressure, and $\lambda=\varepsilon_{Q} /\left(\varepsilon_{N}+\varepsilon_{Q}\right)$ is the density jump parameter.

\section{Summary}

The presence of the $\delta$-meson field leads to the decrease of both transition pressure, $P_{0}$, and baryon number densities $n_{N}$ and $n_{Q}$. Besides, the jump parameter, $\lambda$, is increased. According to Ref.[7], in case of $\lambda>\lambda_{c r}=3 / 2$, the infinitisimal core of the new phase is unstable. Our analysis show that in case of $B<B_{c r}=69.3 \mathrm{MeV} / \mathrm{fm}^{3}$, the density jump parameter satisfies the condition $\lambda>\lambda_{c r}$, and the neutron star configurations with infinetisimal quark cores are unstable.

Acknowledgements. This work is supported by the Ministry of Education and Science of Armenia under project No.130(2008).

\section{References}

[1] J.D. Walecka, Ann. Phys. 83, 491 (1974); B.D. Serot, J.D. Walecka, Int.J.Mod.Phys. E6, 515 (1997).

[2] B. Liu, V. Greco, V. Baran, M. Colonna, M. Di Toro, Phys.Rev. C65, 045201 (2002).

[3] V. Greco, M. Colonna, M. Di Toro, F. Matera, Phys. Rev. C67, 015203 (2003).

[4] N.K. Glendenning, Phys. Rev. D46, 1274 (1992).

[5] G.B. Alaverdyan, Astrophysics 52, 147 (2009).

[6] E. Farhi, R.L. Jaffe, Phys.Rev. D30, 2379 (1984).

[7] Z.N. Seidov, Astron.Zh., 15, 347 (1971). 